\title{
Microscopic hematuria predicts lower stage in patients with upper tract urothelial carcinoma
}

This article was published in the following Dove Press journal:

Cancer Management and Research

\author{
Nienie $Q i^{1, *}$ \\ Jiufeng Zhang2,* \\ Yue Chen' \\ Rumin Wen' \\ Hailong $\mathrm{Li}^{\prime}$ \\ 'Department of Urology, The \\ Affiliated Hospital of Xuzhou \\ Medical University, Xuzhou, China; \\ ${ }^{2}$ Department of Urology, Peking \\ University First Hospital, Institute of \\ Urology, Peking University, National \\ Urological Cancer Center, Beijing, \\ China
}

*These authors contributed equally to this work
Correspondence: Hailong Li; Rumin Wen Department of Urology, The Affiliated Hospital of Xuzhou Medical University, No. 99 Huaihai West Road, Quanshan District, Xuzhou 221100, China Emailwwvivil63@163.com; II0248578I@qq.com
Background: The aim of this study was to assess the association between the severity of hematuria (microscopic or gross) and the tumor stage and grade in a population of histopathologically confirmed upper tract urothelial carcinoma (UTUC) patients.

Patients and methods: We conducted a multicenter, observational study of patients who were newly diagnosed with UTUC between January 2011 and December 2016. Demographic information, pathology, and the status of hematuria were retrospectively reviewed. The association between the severity of hematuria and the tumor stage and grade was evaluated using logistic regression.

Results: The UTUC patients presented with gross hematuria ( $\mathrm{GH}, 76.7 \%)$, microscopic hematuria (MH, 11.1\%), and no hematuria $(12.2 \%)$ at the time of diagnosis. The pathological stages at diagnosis for those with $\mathrm{MH}$ were $\mathrm{Ta}$ in $5.1 \%, \mathrm{~T} 1$ in $47.5 \%$, and $\geq \mathrm{T} 2$ in $47.5 \%$. The stages at diagnosis for those with $\mathrm{GH}$ were $\mathrm{Ta}$ in $1.7 \%$, $\mathrm{T} 1$ in $35.5 \%$, and $\geq \mathrm{T} 2$ in $62.7 \%$. On univariate and multivariate logistic regression analyses, after adjusting for clinical factors such as age, gender, and smoking history, GH was an independent risk factor for muscle-invasive UTUC ( $\geq \mathrm{T} 2$ disease) at diagnosis (OR 1.89, 95\% CI 1.073-3.329; $P=0.027$ ). High-grade tumor was found in $47.8 \%$ of patients with $\mathrm{GH}$ and $39.0 \%$ of those with $\mathrm{MH}$. The severity of hematuria was not associated with tumor grade.

Conclusion: We are the first to report evidence that microscopic hematuria at presentation accurately predicts lower pathological stage in patients with newly diagnosed UTUC. Earlier detection of disease, before the development of GH, may influence the treatment decision and survival. The type of hematuria at the time of diagnosis does not impact the tumor grade.

Keywords: upper tract urothelial carcinoma, gross hematuria, microscopic hematuria, stage

\section{Introduction}

Urothelial carcinomas (UCs) are the fifth most common tumors. ${ }^{1}$ They can be located in the lower or upper urinary tract. Compared to bladder cancer, upper tract UCs (UTUCs) are relatively uncommon and account for only 5\%-10\% of UCs. ${ }^{2}$ UTUC includes renal pelvic carcinoma and ureteral carcinoma. Approximately $60 \%$ of patients with UTUC are invasive at diagnosis. ${ }^{3}$ Radical nephroureterectomy (RNU) with bladder cuff excision is the standard treatment for high-risk UTUC, regardless of tumor location. ${ }^{4}$ However, chronic kidney disease is prevalent after RNU as there is a substantial decline in the estimated glomerular filtration rate. ${ }^{5}$ Upper tract UCs that invade the muscle wall usually have a poor prognosis. The 5 -year specific survival rate is $<50 \%$ for patients with pT2/pT3 tumors and $<10 \%$ for those with pT4. ${ }^{6-8}$ Therefore, earlier detection before invasion and conservative management of small, low-grade, and non-invasive 
UTUCs are important for preserving kidney function and improving survival for patients with UTUC. Currently, UTUC is often detected after the occurrence of gross hematuria $(\mathrm{GH})$ or microscopic hematuria $(\mathrm{MH})$, which is the most common symptom of UTUC $(70 \%-80 \%) .{ }^{9}$ It is often assumed that $\mathrm{MH}$ tends to occur before $\mathrm{GH}$ in the disease development process. However, this has not been fully explicated and it is unknown whether $\mathrm{GH}$ at the time of diagnosis predicts a more invasive disease than $\mathrm{MH}$.

In the present study, we investigated the status of hematuria at presentation to predict the disease stage and tumor grade. To the best of our knowledge, there is no available study regarding the association of the severity or presence/ absence of hematuria and the stage and grade of UTUC patients.

\section{Patients and methods}

We conducted a multicenter, observational cohort study of patients diagnosed with UTUC between January 2011 and December 2016. A total of 532 patients with histologically confirmed UTUC were retrospectively enrolled in the present study. Informed consent was obtained from all the participants. Clinicopathologic parameters were collected from the medical records, including age at initial diagnosis, gender, smoking history, and severity or presence/absence of hematuria at the time of diagnosis, to evaluate their impact on the stage and grade. All of the procedures performed in studies involving human participants were in accordance with the ethical standards of the institutional and national research committee and with the 1964 Helsinki Declaration and its later amendments or comparable ethical standards. This study was approved by the institutional review board of Peking University First Hospital and The Affiliated Hospital of Xuzhou Medical University. All the patients provided written informed consent.

GH was defined as visible blood in the urine reported by the patient, while $\mathrm{MH}$ was defined as more than three red blood cells/high-power field on urine analysis. All the pathological specimens were reexamined by an experienced urological pathologist to confirm the original diagnosis. The tumor stage was assessed according to the UICC 2009 TNM classification of malignant tumors. The tumor grade was assessed according to the 2004 WHO classification grading system.

For statistical analysis, numerical variables were compared using independent $t$-tests and categorical variables were compared using chi-square tests. The association between variables and tumor grade or stage was examined by using univariate and multivariate logistic regression analyses. The stage was categorized as a dichotomous variable based on the presence of invasion ( $\geq \mathrm{T} 2$ vs $\mathrm{T} 1+\mathrm{Ta}$ ). All the statistical analyses were conducted using SPSS version 20.0 (IBM Corporation, Armonk, NY, USA). All the reported $P$-values were two sided, and statistical significance was set at $<0.05$.

\section{Informed consent}

Written informed consent was obtained from the patient for the publication of this report.

\section{Ethical approval}

The study was approved by the institutional review board from Peking University First Hospital and The Affiliated Hospital of Xuzhou Medical University.

\section{Results}

Exactly 532 consecutive UTUC patients were enrolled in the present study and their clinicopathologic characteristics are described in Table 1. There were 273 male and 259 female patients with a mean age of 66.8 years. The diagnosis of UC was pathologically confirmed in all the patients. A total of 214 patients had non-muscle-invasive diseases ( $\leq \mathrm{pT} 1)$ and 318 patients had muscle-invasive diseases ( $\geq \mathrm{pT} 2)$, while 286 patients had low-grade tumors and 246 patients had highgrade tumors. The patients presented with $\mathrm{GH}(408,76.7 \%)$, $\mathrm{MH}(59,11.1 \%)$, and no hematuria $(65,12.2 \%)$. Women were more likely to present with $\mathrm{MH}$ than men $(14.3 \%$ vs $8.1 \%$, $P=0.003)$. Most of the patients with UTUC had no history of smoking, and GH was prevalent in those with a history of smoking.

After excluding the patients with no hematuria, the pathological stages at diagnosis for those with $\mathrm{MH}$ were Ta in 5.1\%, $\mathrm{T} 1$ in $47.5 \%$, and $\geq \mathrm{T} 2$ in $47.5 \%$. The stages at diagnosis for the patients with $\mathrm{GH}$ were $\mathrm{Ta}$ in $1.7 \%$, $\mathrm{T} 1$ in $35.5 \%$, and $\geq \mathrm{T} 2$ in $62.7 \%$. On univariate and multivariate logistic regression analyses, after adjusting for clinical factors such as age, gender, and smoking history, GH was an independent risk factor for muscle-invasive UTUC ( $\geq \mathrm{T} 2$ disease) at diagnosis (OR 1.89 , 95\% CI 1.073-3.329; $P=0.027$ ), as shown in Table 2.

After excluding the patients with no hematuria, highgrade tumor was found in $47.8 \%$ of those with $\mathrm{GH}$ and $39.0 \%$ of those with $\mathrm{MH}$. The severity of hematuria was not associated with the tumor grade. None of other clinical factors predicted the grade of disease.

In the 467 patients who presented with hematuria, the type of hematuria (MH or $\mathrm{GH}$ ) after stratification by both pathological stage and grade is shown in Table 3. MH had 
Table I Clinical and pathological characteristics of UTUC patients according to the type of hematuria

\begin{tabular}{|c|c|c|c|c|c|c|}
\hline \multirow[t]{2}{*}{ Variable } & & \multicolumn{3}{|c|}{ Type of hematuria, n (\%) } & \multirow[t]{2}{*}{ Total, n } & \multirow[t]{2}{*}{$P$-value } \\
\hline & & Gross & Microscopic & None & & \\
\hline Age (mean, years) & & 67.0 & 66.8 & 65.0 & 66.8 & 0.388 \\
\hline \multirow[t]{2}{*}{ Gender } & Male & $226(82.8)$ & $22(8.1)$ & $25(9.2)$ & 273 & 0.003 \\
\hline & Female & $182(70.3)$ & $37(14.3)$ & $40(15.4)$ & 259 & \\
\hline \multirow[t]{2}{*}{ Smoking } & Yes & $102(85.0)$ & $4(3.3)$ & $14(11.7)$ & 120 & 0.007 \\
\hline & No & $306(74.3)$ & $55(13.3)$ & $51(12.4)$ & 412 & \\
\hline \multirow[t]{2}{*}{ Grade } & High & $195(79.3)$ & $23(9.3)$ & $28(11.4)$ & 246 & 0.385 \\
\hline & Low & $213(74.5)$ & $36(12.6)$ & $37(12.9)$ & 286 & \\
\hline \multirow[t]{5}{*}{ Stage } & $\mathrm{Ta}$ & $7(58.3)$ & $3(25.0)$ & $2(16.7)$ & 12 & 0.044 \\
\hline & TI & $145(7 \mid .8)$ & $28(13.9)$ & $29(14.4)$ & 202 & \\
\hline & $\mathrm{T} 2$ & $126(79.7)$ & $14(8.9)$ & $18(11.4)$ & 158 & \\
\hline & T3 & $115(82.1)$ & $12(8.6)$ & $13(9.3)$ & 140 & \\
\hline & $\mathrm{T} 4$ & $15(75.0)$ & $2(10.0)$ & $3(15.0)$ & 20 & \\
\hline
\end{tabular}

Abbreviation: UTUC, upper tract urothelial carcinoma.

Table 2 Multivariate logistic regression analysis of factors predicting high grade or stage $(\geq T 2)$

\begin{tabular}{|l|l|l|l|l|}
\hline & \multicolumn{2}{|l|}{ Stage } & \multicolumn{2}{l|}{ Grade } \\
\hline & OR (95\% CI) & $\boldsymbol{P}$-value & OR (95\% CI) & P-value \\
\hline Age & $0.993(0.976-1.01 \mathrm{I})$ & 0.444 & $1.003(0.986-1.020)$ & 0.753 \\
Gender & $0.837(0.555-1.26 I)$ & 0.394 & $1.253(0.838-1.873)$ & 0.272 \\
Smoking & $0.86 I(0.517-1.434)$ & 0.565 & $0.787(0.483-1.283)$ & 0.336 \\
GH & $1.766(1.011-3.084)$ & 0.046 & $0.695(0.394-1.224)$ & 0.208 \\
\hline
\end{tabular}

Abbreviation: $\mathrm{GH}$, gross hematuria.

Table 3 Positive and negative predictive values of $\mathrm{MH}$ for predicting low-grade and non-muscle-invasive UTUC

\begin{tabular}{|l|l|}
\hline & Hematuria $(\mathbf{n = 4 6 7})$ \\
\hline Non-muscle-invasive cancer $(\mathrm{Ta}+\mathrm{TI})$ & \\
Positive predictive value & $52.5 \%$ \\
Negative predictive value & $62.7 \%$ \\
Low-grade cancer & \\
Positive predictive value & $61.0 \%$ \\
Negative predictive value & $47.8 \%$ \\
\hline
\end{tabular}

Abbreviations: $\mathrm{MH}$, microscopic hematuria; UTUC, upper tract urothelial carcinoma.

a positive predictive value (PPV) and negative predictive value of $52.5 \%$ and $62.7 \%$ for non-muscle-invasive UTUC, respectively. MH had a PPV and negative predictive value of $61.0 \%$ and $47.8 \%$ for low-grade UTUC, respectively.

\section{Discussion}

Currently, UC is often detected on evaluation of patients who present with $\mathrm{GH}$ or $\mathrm{MH}$. Much attention has been paid to determine which patients should or should not be referred to urologists for further assessment. ${ }^{10-12}$ However, the association between the status of hematuria at presentation and the tumor stage or grade is not clear. It is unknown whether presentation with $\mathrm{GH}$ at diagnosis means a worse prognosis than having $\mathrm{MH}$. A previous study reported that local symptoms (including flank/lumbar pain, $\mathrm{GH}$, or a palpable mass) did not confer a worse prognosis compared to patients with incidentally detected UTUC. ${ }^{13}$ Ramirez et al found that GH at the time of diagnosis was associated with a more advanced stage in patients with newly diagnosed bladder cancer. ${ }^{14}$ The association of the UTUC stage or grade at diagnosis with GH vs MH has never been explored.

In our multicenter study, we tried to describe the impact of the severity of hematuria on the stage and grade of UTUC. We found that $\mathrm{MH}$ at diagnosis predicted a lower tumor stage of UTUC in multivariate logistic regression analysis. In fact, there was an $\sim 80 \%$ higher risk for invasive $(\geq \mathrm{pT} 2)$ disease at the time of diagnosis when patients presented with $\mathrm{GH}$. However, patients with low and high grade of disease had a similar distribution of $\mathrm{MH}$ and $\mathrm{GH}$.

The management and survival of UTUC is stage dependent, and patients who are diagnosed when their disease is muscle invasive have a significantly worse survival than those with non-muscle-invasive UTUC. ${ }^{15}$ However, it is difficult to accurately determine the stage of UTUC using currently available methods. Urinary cytology and radiography are unable to accurately predict muscle-invasive disease in UTUC patients. Su et al retrospectively assessed the diagnostic accuracy of fluorescence in situ hybridization (FISH) for detecting aggressive UTUC and found that the overall sensitivity of FISH for detecting advanced UTUC was $62.4 \% .{ }^{16}$ They suggested that voided urine FISH results might predict advanced 
UTUC. Although ureteroscopy is more sensitive and specific for diagnosing UTUC than urine cytology and radiography, it may not be accurate for predicting tumor stage. Straub et al found that the accuracy of preoperative ureteroscopy for predicting the correct tumor grade in UTUC patients was only $58 \%$; this accuracy increased to only $68 \%$ when urinary cytology was also considered. ${ }^{17}$ Furthermore, ureteroscopy is an invasive procedure that requires general anesthesia and involves the accompanying surgical complications, and a recent study indicated that patients were at a higher risk of intravesical recurrence after RNU when they had undergone ureteroscopy before RNU. ${ }^{18}$

Because of the inaccurate preoperative or transurethral staging of UTUC, many RNUs are performed unnecessarily. Preoperative prediction of the pathological stage or patients' prognosis may reduce this overtreatment by selecting patients for nephron-sparing surgery. Diagnostic tools, or combinations of tools, which are effective for determining the UTUC stage are urgently needed to identify patients for whom conservative management is the most appropriate treatment strategy. The present study found that many risk factors associated with the detection of UTUC, including gender and smoking history, were not associated with the stage at presentation. Only $\mathrm{MH}$ at presentation was independently associated with lower stage. Combined with other diagnostic techniques, the severity of hematuria may be helpful for distinguishing tumors best treated using conservative management from those that should be treated with RNU.

Some studies suggested that some patients had low risk of cancer and could avoid extensive evaluations using a stratifying risk of urinary tract malignant tumors in patiens with $\mathrm{MH} .{ }^{19}$ However, the current evidence showed that most patients with $\mathrm{MH}$ were not appropriately referred to an urologist for further evaluation and did not undergo imaging examination..$^{20,21}$ Delays in the diagnosis of UTUC can lead to upstaging of tumor and delay in surgery, which may result in the change of management and worse prognosis. ${ }^{22,23}$ Therefore, the appropriate evaluation of UC in patients with MH may improve the survival of those with UTUC.

Some limitations of our study should be considered when interpreting the results. First, this study is retrospective, with all the attendant imprecisions associated with the large recollection of data. The data were collected in two centers and there were certainly biases related to differences in pathologic assessment between the two centers. In addition, the sample size of the patients with $\mathrm{MH}$ at diagnosis was relatively small. Only $15 \%$ patients with hematuria ( $\mathrm{GH}$ or $\mathrm{MH}$ ) presented with $\mathrm{MH}$, which may influence the reliability of the PPV of $\mathrm{MH}$ for predicting non-muscle-invasive UTUC. Finally, we were unable to evaluate the relationship between the severity of hematuria and oncologic prognosis due to the short follow-up period. Similar studies evaluating the relationship between the status of hematuria and tumor stage of UTUC in additional populations are needed to confirm our findings. Despite these limitations, to the best of our knowledge, this is the first study to provide the correlation between the severity of hematuria and the tumor stage of UTUC.

\section{Conclusion}

We are the first to report evidence that $\mathrm{MH}$ at presentation accurately predicts lower pathological stage in patients with newly diagnosed UTUC. Earlier detection of disease, before the development of $\mathrm{GH}$, may influence the treatment decision and survival. The type of hematuria at the time of diagnosis does not impact the tumor grade.

\section{Data sharing statement}

Please contact the author for data requests.

\section{Acknowledgments}

This work was supported by the National Natural Science Foundation of China (No. 81502193), the Science and Technology Department of Jiangsu Province (No. BK20151167), and Distinguished Talents of Six Domains in Jiangsu Province (No. WSW-064).

\section{Disclosure}

The authors report no conflicts of interest in this work.

\section{References}

1. Siegel RL, Miller KD, Jemal A. Cancer statistics, 2016. CA Cancer J Clin. 2016;66(1):7-30.

2. Munoz JJ, Ellison LM. Upper tract urothelial neoplasms: incidence and survival during the last 2 decades. J Urol. 2000;164(5):1523-1525.

3. Margulis V, Shariat SF, Matin SF, et al. Outcomes of radical nephroureterectomy: a series from the Upper Tract Urothelial Carcinoma Collaboration. Cancer. 2009;115(6):1224-1233.

4. Rouprêt M, Babjuk M, Compérat E, et al. European Association of Urology guidelines on upper urinary tract urothelial carcinoma: 2017 update. Eur Urol. 2018;73(1):111-122.

5. Lane BR, Smith AK, Larson BT, et al. Chronic kidney disease after nephroureterectomy for upper tract urothelial carcinoma and implications for the administration of perioperative chemotherapy. Cancer. 2010;116(12):2967-2973.

6. Abouassaly R, Alibhai SM, Shah N, Timilshina N, Fleshner N, Finelli A. Troubling outcomes from population-level analysis of surgery for upper tract urothelial carcinoma. Urology. 2010;76(4):895-901.

7. Lughezzani G, Burger M, Margulis V, et al. Prognostic factors in upper urinary tract urothelial carcinomas: a comprehensive review of the current literature. Eur Urol. 2012;62(1):100-114.

8. Jeldres C, Sun M, Isbarn H, et al. A population-based assessment of perioperative mortality after nephroureterectomy for upper-tract urothelial carcinoma. Urology. 2010;75(2):315-320.

9. Cowan NC. CT urography for hematuria. Nat Rev Urol. 2012;9(4): 218-226. 
10. Bruyninckx R, Buntinx F, Aertgeerts B, Van Casteren V. The diagnostic value of macroscopic haematuria for the diagnosis of urological cancer in general practice. Br J Gen Pract. 2003;53(486):31-35.

11. Davis R, Jones JS, Barocas DA, et al. Diagnosis, evaluation and followup of asymptomatic microhematuria (AMH) in adults: AUA guideline. J Urol. 2012;188(6 Suppl):2473-2481.

12. Cohen RA, Brown RS. Clinical practice. Microscopic hematuria. $N$ Engl J Med. 2003;348(23):2330-2338.

13. Raman JD, Shariat SF, Karakiewicz PI, et al. Does preoperative symptom classification impact prognosis in patients with clinically localized upper-tract urothelial carcinoma managed by radical nephroureterectomy? Urol Oncol. 2011;29(6):716-723.

14. Ramirez D, Gupta A, Canter D, et al. Microscopic haematuria at time of diagnosis is associated with lower disease stage in patients with newly diagnosed bladder cancer. BJU Int. 2016;117(5):783-786.

15. Mbeutcha A, Rouprêt M, Kamat AM, et al. Prognostic factors and predictive tools for upper tract urothelial carcinoma: a systematic review. World J Urol. 2017;35(3):337-353.

16. $\mathrm{Su} \mathrm{X,} \mathrm{Hao} \mathrm{H,} \mathrm{Li} \mathrm{X,} \mathrm{et} \mathrm{al.} \mathrm{Fluorescence} \mathrm{in} \mathrm{situ} \mathrm{hybridization} \mathrm{status} \mathrm{of}$ voided urine predicts invasive and high-grade upper tract urothelial carcinoma. Oncotarget. 2017;8(16):26106-26111.
17. Straub J, Strittmatter F, Karl A, Stief CG, Tritschler S. Ureterorenoscopic biopsy and urinary cytology according to the 2004 WHO classification underestimate tumor grading in upper urinary tract urothelial carcinoma. Urol Oncol. 2013;31(7):1166-1170.

18. Guo RQ, Hong P, Xiong GY, et al. Impact of ureteroscopy before radical nephroureterectomy for upper tract urothelial carcinomas on oncological outcomes: a meta-analysis. BJU Int. 2018;121(2):184-193.

19. Loo RK, Lieberman SF, Slezak JM, et al. Stratifying risk of urinary tract malignant tumors in patients with asymptomatic microscopic hematuria. Mayo Clin Proc. 2013;88(2):129-138.

20. Buteau A, Seideman CA, Svatek RS, et al. What is evaluation of hematuria by primary care physicians? Use of electronic medical records to assess practice patterns with intermediate follow-up. Urol Oncol. 2014;32(2):128-134.

21. Nieder AM, Lotan Y, Nuss GR, et al. Are patients with hematuria appropriately referred to Urology? A multi-institutional questionnaire based survey. Urol Oncol. 2010;28(5):500-503.

22. Waldert M, Karakiewicz PI, Raman JD, et al. A delay in radical nephroureterectomy can lead to upstaging. BJU Int. 2010;105(6):812-817.

23. Lee JN, Kwon SY, Choi GS, et al. Impact of surgical wait time on oncologic outcomes in upper urinary tract urothelial carcinoma. J Surg Oncol. 2014;110(4):468-475.
Cancer Management and Research

\section{Publish your work in this journal}

Cancer Management and Research is an international, peer-reviewed open access journal focusing on cancer research and the optimal use of preventative and integrated treatment interventions to achieve improved outcomes, enhanced survival and quality of life for the cancer patient. The manuscript management system is completely online and includes

\section{Dovepress}

a very quick and fair peer-review system, which is all easy to use. Visit http://www.dovepress.com/testimonials.php to read real quotes from published authors. 